\title{
La oferta formativa de Relaciones Públicas en los nuevos Grados universitarios en España (2009-10)
}

\author{
The offer of training and education in Public Relations \\ in the new university Degrees in Spain (2009-10)
}

\author{
Dra. Kathy Matilla \\ Universidad Ramón Llull, URL, ES \\ kathyms@blanquerna.url.edu \\ Dr. Salvador Hernández \\ Universidad Católica San Antonio, UCAM, ES \\ SHernandez@pdi.ucam.edu \\ Dr. José Carlos Losada \\ Universidad Católica San Antonio, UCAM, ES \\ jclosada@hotmail.com
}

Recibido: 29 de Septiembre de 2010

Aceptado: 30 de Noviembre de 2010

\begin{abstract}
Resumen
Este artículo pretende exponer la situación de partida de los contenidos curriculares (asignaturas) específicos de Relaciones Públicas de los nuevos grados de Publicidad y Relaciones Públicas del Sistema Universitario español en el curso 2009-10, primer año de la implantación masiva adaptada al Espacio Europeo de Educación Superior (EEES) que permite total libertad de denominación de grados y asignaturas, por medio de un rastreo sistemático de las páginas web de las universidades de la unidad de análisis, cuyo resultado ha consistido en una no visualización de cambios notables con respecto al modelo universitario anterior a la implantación del Proceso de Bolonia (licenciaturas), ni en lo que se refiere a la denominación del título, ni con respecto a una mejora en la histórica paridad desigual de las asignaturas de Relaciones Públicas respecto a las de Publicidad, en clara desventaja de las primeras.
\end{abstract}

\section{Abstract}

This article endeavours to present the initial situation of specific curriculum contents 
(university classes) in Public Relations of the new degrees in Advertising and Public Relations of the Spanish University System in the year 2009-2010, the first year of the massive implementation adapting to the European Space for Higher Education (EEES), which permits total freedom of designation of degrees and classes by means of a systematic search of the web pages of the universities of the unit of analysis, the result of which has been characterized by a lack of notable changes compared to the university model prior to the implementation of the Bologna Process (the previous university diploma system), both regarding the designation of the degree, as well as regarding any improvement in the historically unequal status of the classes in Public Relations compared to those in Advertising, to the clear detriment of the former.

Palabras Clave: Relaciones Públicas; Grado; Asignaturas; Espacio Europeo de Enseñanza Superior (EEES); España; Proceso de Bolonia.

Keywords: Public Relations; degree; university class; European Space for Higher Education; Spain; Bologna Process.

Sumario: 1. Introducción. 1.1. Propósito. 1.2. Objetivos. 1.3. Problema. 1.4. Importancia. 1.5. Situación. 1.6. Contribuciones. 1.7. Hipótesis. 1.8. Justificación. 2. Metodología. 2.1. Método. 2.2. Diseño. 2.3. Población y Muestra. 2.4. Unidad de análisis. 2.5. Instrumento. 2.6. Rigor. 2.7. Validez. 2.8. Razonamiento. 3. Resultados. 4. Hallazgos. 4.1. Credibilidad. 4.2. Discusión. 4.3. Comparación. 4.4. Nuevas investigaciones. 4.5. Conclusiones. 5. Bibliografía.

Summary: 1. Introduction. 1.1. Topic. 1.2. Objectives. 1.3. Problem. 1.4. Importance. 1.5. Situation. 1.6. Contributions. 1.7. Hypothesis. 1.8. Justification. 2. Methodology. 2.1. Method. 2.2. Design. 2.3. Population and sample. 2.4. Unit of analysis. 2.5. Instrument. 2.6. Rigour. 2.7. Validity. 2.8. Reasoning. 3. Results. 4. Discoveries. 4.1. Credibility. 4.2. Discussion. 4.3. Comparison. 4.4. New research. 4.5. Conclusions. 5. Bibliography.

Translation by Catherine Otey (B.A. summa cum laude in Speech and Drama from Trinity University - Universidad Ramon Llull)

\section{Introducción}

\subsection{Propósito}

La investigación que se presenta tiene como propósito fundamental constituirse en el punto de referencia inicial sobre los contenidos curriculares del nuevo grado universitario en Publicidad y Relaciones Públicas adaptado al Espacio Europeo de Educación Superior EEES- (ANECA, 2005), ofertado por el Sistema Universitario del estado español en el curso 
2009-10 y, específicamente, en referencia a las asignaturas del área de conocimiento de las Relaciones Públicas.

La investigación pretende erigirse en el punto de partida que la Asociación de Directivos ADC Dircom Cataluña, desde su "Cátedra Dircom.Cat", precisa para, en los próximos cinco años (2010-2015), establecer un observatorio que permita comprobar las posibles evoluciones y mejoras introducidas en dichos contenidos curriculares, con la finalidad de elaborar un futuro Libro Blanco propio que, desde una parte del ámbito profesional -la de los directivos de Comunicación-, defina claramente las competencias requeridas por la praxis a los graduados y postgraduados universitarios especializados en Relaciones Públicas (Lalueza, 2009) y, en la medida de lo posible, contribuir a mejorar los perfiles profesionales adscritos a este campo de conocimiento y definidos por el "Libro Blanco" de la ANECA para el Título del Grado en Publicidad y Relaciones Públicas: "profesionales que ejercen su actividad desde el ámbito de las organizaciones o desde una agencia o desde la empresa consultora, y profesionales responsables de la gestión estratégica de la Comunicación Corporativa" (ANECA, 2005: 333).

Se trata, en definitiva, de cristalizar en realidad una de las metas básicas del espíritu del EEES (Casado, 2006; Karseth, 2006; Sánchez y Zubillaga, 2005; Sanjosé, 2007; Velasco, 2005):, conocido popularmente como Proceso de Bolonia: tender puentes de diálogo y entendimiento entre la academia y el sector profesional, en línea con otras iniciativas similares desarrolladas en el estado español, como la de la "Comisión de Educación en Relaciones Públicas" de AIRP - Asociación de Investigadores de Relaciones Públicas, en alianza con ADECEC - Asociación de Empresas Consultoras en Relaciones Públicas y Comunicación (Xifra, 2007: 212), adherido a los postulados de la "Commission on Public Relations Education" (2006), descritos en "The Professional Bond - Public Relations Education and the Practice", liderado por la Global Alliance for Public Relations and Communication Management y patrocinado por la Public Relations Society of America Foundation, a cuyos principios y postulados también se ha adscrito la "Cátedra Dircom.Cat".

Conviene recordar que: "The Commission on Public Relations Education is composed of public relations educators and practitioners who represent 12 professional societies in public relations and related fields of communications", y que su informe "The Professional Bond Public Relations Education and the Practice" (2006) constituye un referente internacional de primer orden, cuya actualización será publicada en 2010 (previsión).

\subsection{Objetivo}

Estudiar en profundidad los contenidos curriculares de la oferta de formación de grado del Sistema Universitario español, concretamente en el campo de las Relaciones Públicas y la 
Comunicación Corporativa en el primer año de la implantación masiva de la formación adaptada al Espacio Europeo de Educación Superior (curso 2009-10).

\subsection{Problema}

El problema de fondo quedó claramente expuesto por J. Xifra, (Xifra, 2007: 212), con respecto a los contenidos curriculares del área de Relaciones Públicas de las antiguas licenciaturas (algunos de ellos aún vigentes en las universidades cuyos grados aún no han sido verificados): "The autonomy given to universities allowing them to set their own compulsory and elective contents has clearly undermined public relations, relegating public relations education to second place. Despite its name, the Degree in Advertising and Public Relations does not include two majors. It is an Advertising major with a Public Relations minor".

Lo anteriormente expuesto por Xifra en 2007 configura, a su vez, dos consecuencias de gran calado:

a) Con respecto a la práctica profesional (organizaciones/empresas, consultoras y agencias), la recepción en el ámbito profesional de unos licenciados con una insuficiente preparación académica en la materia en que, presumiblemente, se habían "especializado", es decir, con enseñanzas insuficientes por parte de las universidades objeto de estudio.

b) Con respecto al sistema universitario español, un incumplimiento de contrato para con sus "clientes", los estudiantes universitarios licenciados en Publicidad y Relaciones Públicas, interesados en la especialización en Relaciones Públicas y, asimismo, una flagrante falta de responsabilidad para con el colectivo profesional que debía acogerlos.

\subsection{Importancia}

La gravedad de la situación descrita por Xifra (2007) sobre las antiguas licenciaturas no es tema baladí, sobre todo si se tiene en cuenta que la formación universitaria y su nivel de calidad constituyen un patrimonio fundamental de todo ciudadano. De ahí que el Consejo de Coordinación Universitaria de la Secretaría de Estado de Universidades e Investigación del Ministerio de Educación español, en su informe "Propuestas para la renovación de las metodologías educativas de la universidad" declaraba en 2006 que: "El proceso de construcción del Espacio Europeo de Educación Superior (EEES) se percibe como la oportunidad perfecta para impulsar una reforma que no debe quedarse en una mera reconversión de la estructura y contenidos de los estudios, sino que debe alcanzar al meollo 
de la actividad universitaria, que radica en la interacción profesores-estudiantes para la generación de aprendizaje" (Ministerio de Educación: 2006: 7).

Y también que: "La cuestión de los objetivos y estrategias para la renovación de las metodologías educativas en las universidades españolas requiere de un enfoque integral. Los principales objetivos de un proceso de renovación pedagógica general y generalizada identificados por la Comisión son diez, los cinco primeros de tipo técnico-pedagógico, los segundos de naturaleza político-organizativa: - El fin de la renovación es la mejora de la calidad del aprendizaje. (...) - Es preciso aproximar más los estudios universitarios al ejercicio profesional, potenciando la dimensión práctica de la enseñanza: el saber, sí, pero también el saber hacer y el saber ser/estar".

\subsection{Situación actual}

El Programa Audit (ANECA, 2008) declara que: "El marco EEES y los nuevos cambios introducidos en la normativa española establecen que las universidades deben garantizar en sus actuaciones el cumplimiento de los objetivos asociados a las enseñanzas que imparten, buscando además su mejora continua. Por ello, las universidades deben contar con políticas y Sistemas de Garantía Interna de Calidad (SGIC) formalmente establecidos y públicamente disponibles. De acuerdo a lo anterior, ANECA, en colaboración con las agencias AQU y ACSUG, de forma coordinada, han desarrollado el Programa Audit. Con esta iniciativa dirigida a los Centros Universitarios se pretende orientar el diseño del SGIC que integre las actividades que hasta ahora han venido desarrollándose relacionadas con la garantía de calidad de las enseñanzas".

Con respecto a nuestro objeto de estudio, son 32 las universidades españolas que ofertan titulaciones de Grado/Licenciatura de Publicidad y Relaciones Públicas, según se describirá con mayor detalle más adelante.

Conviene destacar que de estas 32 universidades, un total de 19 (8 públicas y 11 privadas) han visto verificado su Grado (61,3\%), mientras que todavía no lo han conseguido las 13 (10 públicas y 3 privadas) restantes (38,7\%), es decir, que en el curso 2009-10 un total de 19 universidades españolas imparten su oferta formativa adaptada al EEES, en convivencia con otras 13 que aún no han obtenido la verificación del grado, por lo que siguen ofertando el modelo formativo anterior al Proceso de Bolonia (licenciaturas).

\subsubsection{Críticas a la investigación}


De lo anteriormente expuesto deberían desprenderse dos consecuencias inmediatas. La primera de ellas, que debería observarse una mejora notable entre los contenidos curriculares (asignaturas, unidades formativas y módulos) de los grados con respecto a las licenciaturas, cuando menos con respecto a la situación descrita por Xifra en 2007 (pg. 212). La segunda, la imposibilidad de llevar a cabo nuestro estudio de forma homogénea en esta investigación, en tanto no se haya obtenido la verificación de la totalidad de los grados por parte de la totalidad de la unidad de estudio y que, previsiblemente, estimamos que debería estar plenamente cumplimentada el próximo curso académico (2010-11).

Destacamos, asimismo, que en la práctica totalidad de las diversas páginas web consultadas nos ha resultado imposible acceder al detalle de contenidos de las diversas asignaturas, motivo por el cual no hemos podido llevar a cabo un análisis cualitativo de las mismas, en especial de aquellas que comparten su título con respecto a ambas áreas de conocimiento del grado/licenciatura (ej.: "Estrategias de Publicidad y Relaciones Públicas"; "Fundamentos de Publicidad y Relaciones Públicas"; o "Introducción a la Publicidad y las Relaciones Públicas", etc.).

Con respecto a los Grados en Publicidad y Relaciones Públicas estudiados, señalamos carencias informativas importantes en las fuentes consultadas:

1. Hemos comprobado la ausencia casi total de menciones específicas a la estructura de los módulos formativos que engloban los recorridos temáticos de las diversas materias asociadas, y que son característicos del nuevo modelo formativo de los grados adaptados al EEES, ya que en la práctica totalidad de las páginas web consultadas siguen apareciendo las divisiones por cursos (1ำ a $\left.4^{\circ}\right)$ vinculadas al modelo formativo anterior a la implantación del Proceso de Bolonia (licenciaturas), hecho éste que imposibilita al estudiante -potencial o real- una visualización clara de su posible futura especialización mediante sus áreas de elección curricular y, con respecto a nuestro estudio, una comprensión inmediata de la orientación proporcionada en cada centro a los diversos ámbitos de especialización (Publicidad o Relaciones Públicas, o ambas al 50\%).

2. La carencia de información con respecto a la adquisición de las competencias formativas -tanto transversales como específicas- de los estudiantes formalmente vinculadas a cada asignatura del modelo de grado adaptado al EEES es, asimismo, total para cualquier persona que consulte externamente la página web de las universidades/centros analizados.

3. La no presencia de información relativa a las unidades formativas -también denominadas como "grupos pequeños" en algunos centros/universidades- y vinculadas a 
las asignaturas es total o, cuando menos, no nos ha resultado posible acceder a ella sin poseer una clave de acceso como estudiante, PDI o PAS en activo.

4. La única diferenciación con que aparecen presentadas las asignaturas de los nuevos grados en la inmensa mayoría de las páginas web analizadas, además de su división por cursos anteriormente mencionada, consiste en su catalogación como obligatoria/troncal u optativa. Tampoco es mayoritario el número de páginas web objeto de análisis que indiquen qué asignaturas son optativas o que, incluso, las presenten.

5. También constatamos la práctica inexistencia de innovaciones destacables con respecto a las titulaciones, ya que en la gran mayoría de los casos se ha conservado el nombre de la titulación anterior (licenciaturas): "Grado en Publicidad y Relaciones Públicas", incluso en los casos en que se trata de titulaciones propias. La única novedad destacable se detecta en la Universidad Europea de Madrid, UEM y en la Universidad Francisco Varela, UFV (ambas privadas y ubicadas en la Comunidad Autónoma de Madrid), que ofertan, respectivamente, un nuevo grado sólo de Publicidad, en el caso de la UEM, y el denominado de "Publicidad y Experto en nueva Publicidad y Dirección de Eventos Publicitarios", en el caso de la UFV (pese a lo cual mantienen asignaturas de Relaciones Públicas y Comunicación Corporativa).

Confiamos poder observar la subsanación de buena parte de las carencias detectadas en próximas ediciones editoriales de las páginas web analizadas.

\subsection{Contribuciones}

Las principales contribuciones directas identificadas sobre el tema estudiado, tanto nacionales como internacionales, son las aportadas por:

a) La iniciativa llevada a cabo por la "Comisión de Estudios de Relaciones Públicas" de AIRP - Asociación de Investigadores de Relaciones Públicas y su tarea de mejora de la calidad docente vinculada a ADECEC - Asociación de Empresas Consultoras en Relaciones Públicas y Comunicación (Xifra, 2007: 112).

b) El informe de la "Commission on Public Relations Education" (2006) titulado "The Professional Bond - Public Relations Education and the Practice", patrocinado por la norteamericana Public Relations Society of America (Xifra, 2007).

c) El proyecto del Observatorio de la evolución de los contenidos curriculares de los nuevos grados sobre la materia de estudio de la "Cátedra Dircom.Cat" de la delegación territorial 
catalana de la Asociación de Directivos de Comunicación - ADC Dircom (descrito en Hernández; Losada y Matilla, 2009; y en Matilla y Xifra, 2009).

d) El proyecto continuado de ADECEC - Asociación de Empresas Consultoras en Relaciones Públicas y Comunicación consistente en el despliegue bianual del estudio "La Comunicación y las Relaciones Públicas: Radiografía del sector".

e) Los desarrollos editoriales de carácter teórico/práctico de Wright y Turk (2007) "Public Relations Knowledge and Professionalism: Challenges to Educators and Practitioners"; de L'Etang y Pieczka (2006) en "Public Relations Education"; de Botan y Taylor (2004) en "Public Relations: State of the Field"; de Seitel (2001) en "The Practice of Public Relations"; de Tilson y Pérez (2003) en "Public Relations and the New Golden Age of Spain: a Confluence of Democracy, Economic Development and the Media"; de Dozier y Broom (1995) en "Evolution of the Manager Role in Public Relations Practice"; de Broom y Dozier (1986) en "Advancement for Public Relations Role Models", y, finalmente, de Heath y Coombs (2006) en "Today's Public Relations: An Introduction”.

f) La investigación desarrollada por los profesores Huertas y Cavia (2006) "Center and Periphery: Two Speeds for the Implementation of Public Relations in Spain”, publicada en la Public Relations Review, nํ 32.

g) La tesis doctoral de la Dra. Sara Magallón, "Relaciones Públicas: formación y profesión. Definición empírico-operativa del 'practicum' de los estudiantes universitarios de Relaciones Públicas y su vinculación con la realidad profesional en Cataluña, 19921997", defendida en 1998 en la Universidad Autónoma de Barcelona.

De forma secundaria, también consideramos como contribuciones de interés:

h) Las investigaciones realizadas por Matilla y Xifra (2009) y por Hernández; Losada y Matilla, 2009, sobre la oferta formativa de postgrado en Relaciones Públicas que demuestran, respectivamente, la no consideración de la comunicación como área funcional independiente en los contenidos docentes de los masters MBA de las escuelas de negocio españolas y la ausencia de interés de las universidades del estado español por generar oferta de postgrado oficial en el campo de exclusivo conocimiento de las Relaciones Públicas y la Comunicación Corporativa.

i) Las investigaciones llevadas a cabo por Castillo y Xifra (2006) y por Xifra y Castillo (2006) sobre las tesis doctorales en Relaciones Públicas defendidas en el estado español entre 1965 y 2005, que muestran, a través de un “...análisis diacrónico de todas 
las tesis doctorales defendidas públicamente en las universidades españolas como exponente del grado de investigación y de las modalidades y tipologías de los estudios en el contexto de la comunicación en las organizaciones. Consideramos adecuado referirnos a las tesis ya que son uno de los principales referentes de investigación en el campo de la comunicación..." (Castillo y Xifra, 2006: 141), así como que "...la situación en España no es diferente de la de otros países. Aunque el aumento detectado en los últimos años y las perspectivas de futuro son optimistas, compartimos el lamento de Botan y Hazleton [2006] cuando señalan que la escasez de programas de doctorado especializados en relaciones públicas es un problema que afecta al desarrollo de la teoría de las relaciones públicas".

En consecuencia, en España, esta ausencia de programas de doctorado cualificados conlleva un «inconsistente desarrollo de la enseñanza, la teoría y la investigación en relaciones públicas“ (Castillo y Xifra: 2006: 151), pese a lo cual en dichas investigaciones se demuestra cómo, lenta pero paulatinamente, el número de doctores en Relaciones Públicas va nutriendo el tejido universitario de los claustros de las universidades del estado español y, paralela y consecuentemente, incrementando su nivel de excelencia académica.

j) En la misma línea, la aportación de Sallot, Lyon, Acosta-Alzuru, y Jones (2003), "From Aardvark to Zebra: A New Millennium Analysis of Theory Development in Public Relations Academic Journals"; de León Duarte (2007) en "Estrategias y prácticas científicas de las revistas científicas latinoamericanas de la comunicación. Una aproximación a sus características estructurales", y especialmente de Martínez Mendoza (2007) en "En torno a la investigación en Relaciones Públicas", en esta ocasión con respecto a las revistas académicas sobre Relaciones Públicas que permiten la publicación de las investigaciones del personal docente e investigador español.

k) Las diversas descripciones sobre el proceso de convergencia al EEES, la enseñanza basada en las competencias de los estudiantes y los programas de calidad académicodocentes de los autores siguientes: Agut y Lozano (2008); Bará (2008); Caballero (2007); Campillo; Pérez y Gutiérrez (2008); Cano (2008); Escudero; Vallejo y Botías (2008); Esteban y Sáez (2008); Fidalgo y García Sánchez (2007); García Aretio y Ruiz Corbella (2008); García, Gallego y Blanco (2007); García Martínez (2007); Gavari Starkie (2009); González y Raposo (2009); González; Muñoz y Muñoz (2008); Haug (2008); Juanas y Fernández (2008); Martín (2009); Martínez Lirola (2008); Martínez; Martínez y Muñoz (2008); Michavila y Parejo (2008); Michavila y Zamorano (2008); Ortega y Aquillo (2007); Pablos, Colás, González Ramírez y Jiménez Cortés (2007); 
Padilla y Gil (2008); Pardo y García Tobío (2008); Pérez, López, Ureña, Ruiz, Caplloch, González y Castejón (2008); Reques Velasco (2007); Rial (2008); Rodríguez Esteban (2007); Rodríguez Izquierdo (2007); Vázquez (2008); Vázquez (2007); Villa (2008) y Zabalza Beraza (2008).

\subsection{Hipótesis}

El gran esfuerzo que está suponiendo para el sistema universitario español el proceso de convergencia al nuevo modelo formativo del Espacio Europeo de Enseñanza Superior debería verse reflejado en cambios sustanciales en los programas de enseñanza de sus nuevos grados verificados, en especial si se tiene en cuenta la libertad de elección que se abre como nueva opción, en contraposición al encorsetamiento de obligado cumplimiento que imponía el Ministerio de Educación en el modelo anterior (licenciaturas).

Somos conscientes de las dificultades de todo tipo que implica un cambio tan radical como el que nos ocupa, en especial para un sistema sumamente rígido como el que impera en el estado español, poco dado y acostumbrado, por otra parte, a introducir innovaciones. A ello cabe añadir las oportunidades que ofrece la implantación de las nuevas políticas de calidad basadas en el Sistema de Garantía Interna de la Calidad (SGIC), de responsabilidad exclusiva de los centros $-\mathrm{y}$ no de las universidades- para introducir elementos de mejora académico-docente constante por medio del Programa Audit.

Nos encontramos en la etapa inicial del proceso (curso 2009-10) y, por ello, según ha quedado expuesto anteriormente, aún queda mucho por hacer $y$, una vez superado en su totalidad el proceso de verificación de todos los grados, la apuesta por la excelencia formativa basada en las políticas de mejora de la calidad supone la siguiente etapa, que deberá ser permanente. Todo hace prever, pues, que los resultados del cambio deberían verse conforme avance el tiempo.

Todo ello nos lleva a establecer la verificación o refutación de los siguientes planteamientos previos de hipótesis de nuestro trabajo de campo:

P1: ¿Son mayoría (>50\%) los centros de enseñanza universitaria del estado español que ofertan conocimientos en Publicidad y Relaciones Públicas que han obtenido la verificación del grado en el curso 2009-10?

H1: Son mayoría (>50\%) los grados que ofertan conocimientos en Publicidad y Relaciones Públicas que presentan modificaciones en la denominación del mismo en el curso 200910. 
H2: Son mayoría (>50\%) los grados que ofertan conocimientos en Publicidad y Relaciones Públicas que han igualado el número de asignaturas de ambas áreas de conocimiento en el curso 2009-10.

\subsection{Justificación}

Tres años atrás, en un artículo titulado "Undergraduate Public Relations education in Spain: Endangered species?" publicado en Public Relations Review, el Dr. Xifra (2007: 207) afirmaba lo siguiente: "The purpose of this article is to analyze the precarious situation concerning undergraduate public relations education in Spain, despite the existence of a Degree in Advertising and Public Relations. In addition to core subjects, which are set by the government and required on all university curricula, the Spanish system allows universities to complete their curriculum with other compulsory and elective courses. Given this freedom, universities have failed to come up with either the specific contents for public relations or for liberal arts, social sciences and business courses that will enable students to enter the profession. In light of the future restructuring of degrees through the Bologna Process, this situation is a serious threat to the continuity of public relations teaching in Spanish universities (...) So, although the Spanish system differs from the Anglo-Saxon one, the government has set up undergraduate public relations education as a major-one of the two main content, alongside advertising, as a degree that is official nationwide".

Si a lo expuesto se añade que "(...) in addition to setting the contents of degrees according to the skills students are expected to acquire, Spain has decided to eliminate state regulation and increase universities' autonomy and flexibility in determining their own courses. Each university will henceforth be free to decide upon the degrees and contents it so desires. Additionally, the Spanish government has decided to delay adaptation to the European area of higher education until the very last minute (2010), which should be enough time to ensure the survival of public relations education within the new context." (Xifra, 2007: 207), que "Public Relations is the invisible subject in Spanish universities" (Xifra, 2007: 212), y que "Spain suffers from a universal irony: "only a very small number of the nation's senior-level public relations executives and managers studied Public Relations at a university" (Wright \& Turk, 2007, p. 582)" (Xifra 2007: 212), se justifica que, con nuestra investigación, intentemos verificar si, en España, el nuevo sistema universitario derivado del Proceso de Bolonia:

a) va a seguir acogiendo en su seno la formación específica en Relaciones Públicas, y si

b) en caso afirmativo, ésta va a ser de calidad suficiente para integrar con las competencias requeridas a sus futuros graduados en el colectivo profesional especializado en la 
materia que, hoy por hoy, constituye una realidad consolidada, y al cual los centros universitarios, según exige el SGIC, deben rendir cuentas.

\section{Metodología}

\subsection{Método}

El método empleado fue un análisis sistemático por medio de un rastreo de las páginas web de todas las universidades oficiales reconocidas por el Ministerio de Educación del estado español, localizando las ofertas de grados/licenciaturas del curso 2009-10 que pudieran constituirse como unidades de análisis para nuestro estudio.

\subsection{Diseño}

Una vez localizada la oferta, a partir de los requisitos apuntados, se pasó una plantilla de análisis que contemplaba las siguientes variables:

1. Nombre de la Universidad

2. Nombre de la Facultad

3. Localización geográfica

3.1. Ciudad

3.2. Comunidad Autónoma

4. Tipología de Universidad

4.1. Pública / Privada

4.2. Presencial / No presencial (on-line)

5. Grado / Licenciatura

6. Denominación del Grado / Licenciatura

7. Denominación de las asignaturas

7.1. Sólo de Relaciones Públicas

7.2. Sólo de Publicidad

7.3. Mixtas de Relaciones Públicas y Publicidad

8. Clasificación de las asignaturas:

8.1. No optativas

8.2. Optativas

9. Curso en que se imparten las asignaturas

\subsection{Población y muestra}


La población analizada consiste en la totalidad de las universidades que componen el Sistema Universitario del estado español (Ministerio de Educación, 2009: 4) que, en 2009, está configurado por 77 universidades, de las cuales 50 son públicas y 27 privadas.

Del total de dichas 77 universidades, 5 universidades (una pública y cuatro privadas) son universidades no presenciales y 2 son universidades especiales, que únicamente imparten programas especializados de postgrado (Máster y Doctorado): la Universidad Internacional Menéndez Pelayo y la Internacional de Andalucía.

\subsection{Unidad de análisis}

Consideramos como unidad de análisis las universidades españolas del total de 77 , tanto públicas como privadas, presenciales y no presenciales, que ofertan enseñanzas del nuevo Grado de Publicidad y Relaciones Públicas adaptado al Espacio Europeo de Educación Superior o, en su defecto, de la antigua licenciatura, caso de no contar aún con la verificación del título de grado universitario.

Se excluyen de nuestra unidad de análisis las 2 universidades especiales que no imparten grados (Internacional Menéndez Pelayo e Internacional de Andalucía) que, por tal motivo, queda configurada por un total de 75 universidades.

En el curso 2008-09 se verificaron por primera vez 163 nuevos grados universitarios adaptados al EEES, y entre el año 2008 y 2009 se han verificado 1.275 grados. En el curso 2007-08, el último previo a la implantación de los nuevos grados en el curso 2008-09, se adaptaron el $47,8 \%$ de las titulaciones.

La rama de conocimiento en que se ofertan las titulaciones de Grado de Publicidad y Relaciones Públicas es la de Ciencias Sociales y Jurídicas, con el $50 \%$ de sus titulaciones verificadas en el curso 2009-10. Esta rama de conocimiento, en valores absolutos, es la que cuenta con el mayor número de títulos verificados del Sistema Universitario español: 510 en total en 2009 (Ministerio de Educación, 2009: 6).

La unidad de análisis objeto de nuestra investigación está configurada por 31 universidades presenciales (de las cuales 16 son públicas y 15 son privadas), más 1 pública no presencial, lo que supone un total de 32 universidades españolas que ofertan titulaciones de Grado/Licenciatura de Publicidad y Relaciones Públicas, según se indica a continuación, distribuidas por comunidades autónomas y con especificación de si cuentan con oferta formativa verificada (grado adaptado al EEES): 
a) Universidades presenciales

- Públicas [16]

- Cataluña [5]

- Universidad Autónoma de Barcelona, UAB

Grado adaptado al EEES: No

- Universidad de Barcelona, UB

Grado adaptado al EEES: Sí

- Universidad de Gerona, UdG

Grado adaptado al EEES: Sí

- Universidad Pompeu Fabra, UPF

Grado adaptado al EEES: Sí

- Universidad Rovira i Virgili, URV

Grado adaptado al EEES: Sí

- Castilla-León [1]

- Universidad de Valladolid, UVA

Grado adaptado al EEES: No

- Galicia [1]

- Universidad de Vigo, UVI

Grado adaptado al EEES: No

- Andalucía [2]

- Universidad de Sevilla, US

Grado adaptado al EEES: No

- Universidad de Málaga, UMA

Grado adaptado al EEES: No

- Universidad de Cádiz, UCA

Grado adaptado al EEES: No

- País Vasco [1] 
- Universidad del País Vasco, UPV

Grado adaptado al EEES: No

- Madrid [2]

- Universidad Complutense de Madrid, UCM

Grado adaptado al EEES: No

- Universidad Rey Juan Carlos I

Grado adaptado al EEES: Sí

- Murcia [1]

- Universidad de Murcia, UM

Grado adaptado al EEES: No

- Levante [2]

- Universidad Jaume I, UJI

Grado adaptado al EEES: Sí

- Universidad de Alicante, UNA

Grado adaptado al EEES: No

- Privadas [14]

- Cataluña [4]

- Universidad Abad Oliva-CEU, UAO-CEU

Grado adaptado al EEES: No

- Universidad Internacional de Cataluña, UIC

Grado adaptado al EEES: Sí

- Universidad Ramón Llull, UR

Grado adaptado al EEES: Sí

- Universidad de Vic, uVIC

Grado adaptado al EEES: Sí

- Castilla-León [2]

- Universidad Europea Miguel de Cervantes, UOMC

Grado adaptado al EEES: Sí 
- Universidad Pontificia de Salamanca, UPSA

Grado adaptado al EEES: No

- Navarra [1]

- Universidad de Navarra, UNA

Grado adaptado al EEES: Sí

○ Aragón [1]

- Universidad San Jorge, USJ

Grado adaptado al EEES: Sí

- Madrid [5]

- Universidad Antonio de Nebrija, UAN

Grado adaptado al EEES: Sí

- Universidad Europea de Madrid, UEM

Grado adaptado al EEES: Sí

- Universidad Francisco de Vitoria, UFV Grado adaptado al EEES: Sí

- Universidad Camilo José Cela, UCJC

Grado adaptado al EEES: No

- Universidad San Pablo-CEU

Grado adaptado al EEES: Sí

○ Murcia [1]

- Universidad Católica San Antonio, UCAM

Grado adaptado al EEES: Sí

○ Levante [1]

- Universidad Cardenal Herrera Oria-CEU, UCHO-CEU

Grado adaptado al EEES: Sí

b) Universidades no presenciales

- Públicas [1]: 
- Cataluña [1]

- Universitat Oberta de Cataluña, UOC

Grado adaptado al EEES: Sí

De estas 32 universidades, un total de 19 (7 públicas y 12 privadas) han verificado su Grado $(59,4 \%)$, mientras que todavía no lo han conseguido $(40,6 \%)$ las 13 restantes (10 públicas y 3 privadas).

\subsection{Instrumento}

La búsqueda de la oferta formativa del Grado de Publicidad y Relaciones Públicas se ha realizado a través del buscador disponible en la página web del Ministerio de Educación español:

https://www.educacion.es/ructweb/isp/menuDo.do?rama=431001\&tipoEns=CICLO\&pintaCca $\underline{\mathrm{a}=\& \operatorname{cod} A u t=00 \& \operatorname{codProv}=00}$

durante el periodo que abarca el primer trimestre del curso 2009-10 en España (entre el 15 de Septiembre de 2010 y el 31 de Diciembre de 2009).

\subsection{Rigor científico}

Se han revisado los resultados del análisis sistemático de las páginas web de las universidades objeto de estudio en tres ocasiones, con la finalidad de verificar la fiabilidad de la información de interés trasladada a la plantilla creada 'ad hoc'.

Durante este periodo hemos comprobado que se han producido algunos pequeños cambios entre la información publicada en los meses de Septiembre y Octubre de 2009 y la aparecida entre Noviembre y Diciembre de 2010. Dichos cambios afectaban concretamente a 2 universidades de Cataluña y consistieron en un cambio de criterio en la presentación de los programas de estudio de los grados: en la primera versión se presentaban los Módulos, en lugar de las asignaturas por cursos características de las licenciaturas. En la segunda versión, la presentación se realizó siguiendo esta segunda opción, que por otra parte era la adoptada por el resto de las universidades del territorio español.

Muy a nuestro pesar, debemos manifestar que la rigurosidad científica de nuestro trabajo de campo no es total, ya que algunas universidades que ofertan grado no han presentado en sus respectivas páginas web la totalidad de las asignaturas, limitándose a consignar las que 
estaban ofertando en el curso 2009-10 (1ำ y $2^{\circ}$ cursos exclusivamente, según su incorporación al EEES en 2008-09 ó en 2009-10).

\subsection{Validez científica}

La validez científica del estudio rige exclusivamente para el periodo indicado, es decir, el curso 2009-10 y aplicando los datos actualizados con fecha 31.12.09.

\subsection{Razonamiento}

Estimamos que la presentación de los programas docentes en las páginas web de las universidades objeto de estudio analizadas sufrirán más modificaciones los próximos meses. La primera de ellas probablemente sea un ligero cambio en los currículos académicos, conforme los Departamentos vayan llenando de detalle de contenidos las diversas asignaturas que los configuran. El segundo cambio, de mayores consecuencias estructurales en las páginas web, prevemos que se producirá conforme la sociedad vaya comprendiendo la estructura del nuevo sistema educativo universitario, basado en módulos y en competencias de los estudiantes. Confiamos que, en ese momento, los modos/criterios de presentación se modifiquen y aparezca no solamente dicha información, sino, asimismo, la de las competencias y la de las unidades formativas ('grupos pequeños') asociadas a cada asignatura.

\section{Resultados}

\subsection{Nombre de la Facultad}

Hemos constatado que no todos los grados/licenciaturas analizados se imparten en facultades de Comunicación o de Ciencias de la Comunicación: de las 32 universidades objeto de estudio, 14 de ellas $(43,75 \%)$ lo hacen en las que se denominan del modo siguiente:

a) Facultad de Ciencias Sociales (Universidad Abad Oliba-CEU)

b) Facultad de Turismo (Universidad de Gerona)

c) Facultad de Letras (Universidad Rovira i Virgili)

d) Facultad de Empresa y Comunicación (Universidad de Vic)

e) Facultad de Ciencias Humanas e Información (Universidad Europea Miguel de Cervantes)

f) Facultad de Ciencias Sociales, Jurídicas y de la Información (Universidad de Valladolid) 
g) Facultad de Ciencias Sociales y de la Comunicación (Universidad de Vigo; Universidad de Cádiz)

h) Facultad de Ciencias Sociales y Jurídicas (Universidad del País Vasco)

i) Facultad de Ciencias de la Información (Universidad Complutense de Madrid)

j) Facultad de Comunicación y Humanidades (Universidad Europea de Madrid)

k) Facultad de Humanidades y Ciencias de la Comunicación (Universidad San Pablo-CEU; Universidad Cardenal Herrera Oria-CEU)

I) Facultad de Comunicación y Comunicación (Universidad de Murcia)

m) Facultad de Ciencias Humanas y Sociales (Universidad Jaime I)

n) Facultad de Comunicación y Psicología Social (Universidad de Alicante)

\subsection{Localización geográfica}

\subsubsection{Por Comunidades Autónomas}

Del total de 17 comunidades autónomas -además de Ceuta y Melilla- que configuran el estado español, únicamente se imparten conocimientos universitarios en Publicidad y Relaciones Públicas en 10 comunidades autónomas $(58,82 \%)$, todas ellas ubicadas en la península:

- Cataluña (10 universidades)

- Castilla-León (3 universidades)

- Galicia (1 universidad)

- Navarra (1 universidad)

- Aragón (1 universidad)

- Andalucía (3 universidades)

- País Vasco (1 universidad)

- Madrid (7 universidades)

- Región de Murcia (2 universidades)

- Levante (3 universidades)

\subsection{Tipología de Universidad: Pública / Privada - Presencial / No presencial}

- Universidades presenciales: 31

- Públicas: 16

- Privadas: 14

- Universidades no presenciales (on-line): 1

○ Públicas: 1 
- Privadas: 0

\subsection{Grado / Licenciatura}

De las 32 universidades analizadas:

- Han verificado su grado un total de 19 (59,4\% del total)

○ 7 públicas

- 12 privadas

- Todavía no lo han conseguido -o no lo han solicitado- y siguen impartiendo titulación de Licenciatura las 13 restantes $(40,6 \%$ del total)

- 10 públicas

- 3 privadas

\subsection{Denominación del Grado / Licenciatura}

\subsubsection{Grados}

3.5.1.1. Denominación “Grado en Publicidad y Relaciones Públicas" ( $13=40,7 \%$ del total de las 32 universidades analizadas)

- Universidad de Barcelona

- Universidad de Gerona

- Universidad Internacional de Cataluña

- Universidad Pompeu Fabra

- Universidad Ramón Llull

- Universidad Rovira i Virgili

- Universidad de Vic

- Universidad Europea Miguel de Cervantes

- Universidad de Navarra

- Universidad San Jorge

- Universidad Rey Juan Carlos

- Universidad Católica San Antonio

- Universidad Cardenal Herrera Oria-CEU

3.5.1.2. Otras denominaciones de Grados $(7=21,87 \%$ del total de las 32 universidades analizadas)

\subsection{Denominación "Grado en Comunicación"}


- Universitat Oberta de Cataluña

3.5.1.2.2. Denominación "Grado en Publicidad” + "Diploma de especialización en Relaciones Públicas y Dirección de Comunicación” (2 años: $3^{\circ}$ y 4ํㅜ)

- Universidad Antonio de Nebrija

\subsection{Denominación "Grado en Publicidad"}

- Universidad Europea de Madrid

3.5.1.2.4. Denominación “Doble Grado en Humanidades y Publicidad y Relaciones Públicas"

- Universidad San Pablo-CEU

3.5.1.2.5. Denominación “Doble Grado en Periodismo y Publicidad y Relaciones Públicas"

- Universidad San Pablo-CEU

- Universidad Cardenal Herrera Oria-CEU

3.5.1.2.6. Denominación "Doble Grado en Comunicación Audiovisual y Publicidad y Relaciones Públicas"

- Universidad San Pablo CEU (*)

- Universidad Cardenal Herrera Oria-CEU

3.5.1.2.7. Denominación "Grado en Publicidad y Experto en nueva Publicidad y Dirección de Eventos Publicitarios"

- Universidad Francisco de Vitoria

\subsubsection{Licenciaturas}

3.5.2.1. Denominación "Licenciatura en Publicidad y Relaciones Públicas" ( $12=37,5 \%$ del total de las 32 universidades analizadas)

- Universidad Autónoma de Barcelona

- Universidad Pontificia de Salamanca

- Universidad de Valladolid

- Universidad de Vigo

- Universidad de Sevilla

- Universidad de Málaga

- Universidad de Cádiz

- Universidad del País Vasco 
- Universidad Complutense de Madrid

- Universidad Camilo José Cela

- Universidad de Murcia

- Universidad de Alicante

3.5.2.2. Otras denominaciones de Licenciaturas $(2=6,25 \%$ del total de las 32 universidades analizadas)

\subsubsection{Denominación “Doble Licenciatura en Publicidad y Relaciones Públicas y en Periodismo" \\ - Universidad Abad Oliva-CEU}

\subsection{Denominación "Doble Licenciatura en Administración de Empresas y en Publicidad y Relaciones Públicas" \\ - Universidad Abad Oliba-CEU}

\subsection{Denominación de las asignaturas}

Para los fines de nuestra investigación solamente hemos considerado aquellas asignaturas específicas de las áreas de conocimiento de la Publicidad, de las Relaciones Públicas, o de ambas conjuntamente, cuyo total asciende a 732. Se han obviado las de carácter generalista (ej.: Historia del Pensamiento, etc.).

Dadas las dificultades consignadas en el capítulo 2.6. "Rigurosidad científica", nos hemos visto obligados a plantear el trabajo de campo únicamente desde una perspectiva cuantitativa y no cualitativa.

\subsubsection{Asignaturas sólo de Relaciones Públicas}

Se han consignado 140 asignaturas específicas (ej.: "Estrategias de Comunicación Corporativa", "Protocolo", etc.), lo que supone un 19,12\% del total de asignaturas identificadas.

\subsubsection{Asignaturas sólo de Publicidad}

El número de asignaturas con contenidos exclusivamente publicitarios asciende a 449 (ej.: "Dirección de Arte"; "Planificación de Medios"; "Redacción Publicitaria", etc.), es decir, un $61,34 \%$ del total objeto de estudio. 


\subsubsection{Asignaturas mixtas de Relaciones Públicas y de Publicidad}

Las asignaturas conjuntas suman un total de 143 (ej.: "Historia de la Publicidad y de las Relaciones Públicas", "Introducción a la Publicidad y las Relaciones Públicas", etc.), lo que equivale a un $19,54 \%$ del total identificado.

\subsection{Curso en que se imparten las asignaturas}

Dado el carácter cuantitativo de nuestro estudio y a la estructuración en módulos y en créditos europeos (créditos ECTS) de los nuevos grados, esta información debería ser irrelevante, si bien la hemos consignado a efectos de futuros posibles análisis cualitativos.

\section{Hallazgos}

- El hecho de que no todos los grados/licenciaturas analizados de las 32 universidades objeto de estudio se impartan en facultades de Comunicación o de Ciencias de la Comunicación y que 14 de ellas $(43,75 \%)$ se denominen de muy diversas maneras, parece ser un indicador del nivel de confusión y del grado de indeterminación que presenta la oferta universitaria española en lo que se refiere a las áreas de conocimiento de la Publicidad y de las Relaciones Públicas.

- Del total de 17 comunidades autónomas -además de Ceuta y Melilla- que configuran el estado español, únicamente se imparten conocimientos universitarios en Publicidad y Relaciones Públicas en 10 comunidades autónomas $(58,82 \%)$, todas ellas ubicadas en la península. Encabeza el 'ranking' Cataluña, con 10 universidades, seguida de Madrid (7 universidades), Castilla-León, Andalucía y Levante (3 universidades cada una de ellas) y Murcia (2). En las restantes comunidades autónomas analizadas se consigna una única universidad ofertante de la materia objeto de estudio (Galicia, Navarra, Aragón y País Vasco).

Asimismo se constata una clara preeminencia de las universidades pertenecientes a comunidades autónomas ubicadas en la periferia peninsular que ofertan las materias objeto de estudio (Cataluña, Galicia, Andalucía, País Vasco, Murcia y Levante), con respecto a las que se encuentran en el interior del territorio español (Castilla-León, Navarra, Aragón y Madrid). No hay presencia de dicha oferta en Baleares ni en Canarias.

- La unidad de análisis objeto de nuestra investigación está configurada por 31 universidades presenciales (de las cuales 16 son públicas y 15 son privadas), más 1 
pública no presencial, lo que supone un total de 32 universidades españolas que ofertan titulaciones de Grado/Licenciatura de Publicidad y Relaciones Públicas.

- De las 32 universidades analizadas han verificado su grado un total de $19(59,4 \%$ del total), de las cuales 7 son públicas y 12 son privadas. Todavía no lo han conseguido y siguen impartiendo titulación de Licenciatura las 13 restantes (40,6\% del total): 10 públicas y 3 privadas.

- Una vez alcanzada la fecha límite para la implantación de los nuevos grados adaptados al EEES (curso 2009-10), se mantiene la denominación de "Grado en Publicidad y Relaciones Públicas" en 13 universidades $(40,7 \%$ del total de las 32 universidades analizadas), mientras que 7 reciben otras denominaciones diferentes $(21,87 \%$ del total de las 32 universidades analizadas).

- Con respecto a las licenciaturas, reciben la denominación "Licenciatura en Publicidad y Relaciones Públicas" un total de 12 (37,5\% del total de las 32 universidades analizadas) y reciben otras denominaciones diferentes tan sólo 2 de ellas $(6,25 \%$ del total de las 32 universidades analizadas).

- En lo que hace relación a las asignaturas específicas de las áreas de conocimiento de la Publicidad, de las Relaciones Públicas, o de ambas conjuntamente, se han identificado un total de 732, de las cuales 140 son específicas de las Relaciones Públicas (19,12\%); 449 lo son de Publicidad (61,34\%) y 143 son mixtas $(19,54 \%)$.

- En la inmensa mayoría de las universidades analizadas, tanto públicas como privadas, se observa una carencia sistemática de asignaturas sobre técnicas de Relaciones Públicas (ej.: Comunicación de Crisis; 'Lobbying'; Comunicación Interna; etc.), mientras que en las de Publicidad las posibilidades de elección abarcan todo tipo de técnicas (ej.: Planificación de Medios, Redacción Publicitaria y Dirección de Arte) y de posibles subespecializaciones (ej.: Acciones de Publicidad 'Below-the-Line'; Cultura Contemporánea y Publicidad; Música y Publicidad; Nuevas Tecnologías aplicadas a la Publicidad; Sujetos de la actividad publicitaria; Documentación Informativa en Publicidad, etc.). Lo descrito es extensivo tanto a los grados, como a las licenciaturas y las dobles licenciaturas.

- Únicamente el Grado de Publicidad y Relaciones Públicas de la Universidad Cardenal Herrera Oria-CEU (campus de Moncada, Valencia) ha igualado en 5 las asignaturas del área de Relaciones Públicas y las del área de Publicidad y casi lo consigue la Escuela 
Superior de Relaciones Públicas-ESRP, centro adscrito a la Universidad de Barcelona que imparte el Grado en Publicidad y Relaciones Públicas ( 9 de Relaciones Públicas contra 10 de Publicidad).

En contraposición, los casos de falta de paridad más flagrantes son la Licenciatura en Publicidad y Relaciones Públicas de la Universidad de Alicante ( 9 asignaturas de Relaciones Públicas contra 53 de Publicidad); la Licenciatura en Publicidad y Relaciones Públicas de la Universidad Complutense de Madrid (8 vs 30); la Licenciatura en Publicidad y Relaciones Públicas de la Universidad del País Vasco (1 vs 12); la Licenciatura en Publicidad y Relaciones Públicas de la Universidad de Sevilla (4 vs 20); la Licenciatura en Publicidad y Relaciones Públicas de la Universidad de Valladolid (4 vs 16) y la Licenciatura en Publicidad y Relaciones Públicas de la Universidad Autónoma de Barcelona (6 vs 28).

- Hemos elaborado nuestra plantilla consignando los grados/licenciaturas diferenciados por universidades. Observamos que la institución CEU (universidad privada) posee presencia en diversas comunidades autónomas, recibiendo cada universidad una denominación diferente en cada comunidad autónoma:

o Licenciatura en Publicidad y Relaciones Públicas - Universidad Abad Oliva-CEU (Cataluña).

- Doble Grado en Humanidades y en Publicidad y Relaciones Públicas - Universidad San Pablo-CEU (Madrid).

- Grado en Publicidad y Relaciones Públicas - Universidad Cardenal Herrera Oria-CEU (Levante).

- Algunas incongruencias terminológicas, conceptuales y epistemológicas identificadas:

- En la Universidad Antonio de Nebrija, que oferta el "Grado en Publicidad" (sin Relaciones Públicas), aparecen las asignaturas "Principios de Relaciones Públicas" y "Técnicas de las Relaciones Públicas".

○ En el "Grado en Publicidad" (sin Relaciones Públicas) de la Universidad Europea de Madrid aparecen las asignaturas siguientes: "Comunicación Corporativa"; "Gestión Estratégica de las Relaciones Públicas" y "Gestión de Eventos y Protocolo Empresarial". 
- En la Universidad Francisco de Vitoria, en el "Grado en Publicidad y Experto en Nueva Publicidad y Dirección de Eventos Publicitarios" se ofertan, entre otras, las asignaturas: "Estrategias de Comunicación Corporativa"; "Técnicas de Protocolo"; "Protocolo Institucional” y "Técnicas de Comunicación Corporativa.

\subsection{Credibilidad}

La credibilidad de nuestro trabajo de campo entendemos que es total, toda vez que las fuentes a las que hemos acudido -las páginas web de las propias universidades españolas que difunden en ellas sus programas docentes- han sido elaboradas por los propios centros objeto de estudio y, por consiguiente, la fiabilidad de los datos obtenidos es del cien por cien.

\subsection{Discusión}

En el primer año de implantación masiva de los nuevos grados en materia de Publicidad y Relaciones Públicas no se visualizan cambios notables con respecto al modelo anterior (licenciaturas) pese a la libertad que supone el recién estrenado marco del EEES.

De las 732 asignaturas identificadas en Grados y Licenciaturas en el curso 2009-10, 140 son específicas de las Relaciones Públicas (19,12\%); 449 lo son de Publicidad (61,34\%) y 143 son mixtas $(19,54 \%)$, es decir, que las asignaturas específicas del área de Relaciones Públicas siguen teniendo una presencia claramente inferior con respecto a las específicas de Publicidad, tanto en universidades públicas, como en privadas.

Además, dado que es ínfimo el número de universidades que ofertan asignaturas específicas sobre técnicas de Relaciones Públicas, todo parece apuntar a que éstas se desarrollan exclusivamente en asignaturas del tipo "Técnicas de Relaciones Públicas" o "Sistemas y Procesos de las Relaciones Públicas", y ello cuando estas asignaturas existen y no son de carácter mucho más general e introductorio, como por ejemplo "Técnicas de Publicidad y Relaciones Públicas", o "Sistemas y Procesos de la Publicidad y las Relaciones Públicas".

Las incongruencias terminológicas, conceptuales y epistemológicas identificadas en la oferta de asignaturas de los grados de las universidades Antonio de Nebrija, Europea de Madrid y Francisco de Vitoria conducen inexorablemente a interrogarse sobre los criterios adoptados por la Agencia Nacional de Evaluación de la Calidad y Acreditación - Aneca (y por las agencias autonómicas: Agencia Andaluza de Evaluación; Agència de Qualitat Universitària de les Illes Balears; Agencia Canaria de Evaluación y Acreditacion Universitaria; Agencia de Calidad Universitaria de Castilla-La Mancha; Agencia para la Calidad del Sistema Universitario de Castilla-León; Agència per a la Qualitat del Sistema Universitari de 
Catalunya; Axencia para a Calidade do Sistema Universitario de Galicia; y Agència Valenciana d'Avaluació i Prospectiva) para verificar la adaptación de dichos grados al EEES, del mismo modo que tampoco acaba de comprenderse el motivo por el cual han acreditado grados en Publicidad y Relaciones Públicas en los que el segundo ámbito de conocimiento se encuentra en una manifiesta inferioridad de condiciones con respecto al primero.

A fecha de hoy (Diciembre de 2009), no todos los centros universitarios de la muestra objeto de estudio han obtenido la acreditación de sus Sistemas de Garantía Interna de la Calidad por medio del Programa Audit de la Aneca. En su primera convocatoria únicamente han recibido el certificado Audit un total de 117 centros, correspondientes a 49 universidades (web de ANECA descargada el 31.12.09). Cuando ese momento llegue, convendrá analizar, en una posible futura investigación, qué opinan sus 'stakeholders' al respecto de lo expuesto: sus estudiantes, sus graduados, la sociedad y los colectivos profesionales (DirCom, ADECEC y colegios profesionales), a todos los cuales, según la normativa del SGIC, los centros deben rendir cuentas. Y qué mejoras introducen para hacer realidad las políticas de calidad y de transparencia permanentes a las cuales se hayan comprometido.

\subsection{Comparación}

Con respecto a la investigación del profesor Xifra (2007) no se observan en la nuestra mejoras sustanciales dignas de mención tras la fecha límite de adaptación al EEES por parte de las mismas unidades de análisis: las Relaciones Públicas siguen siendo minoritarias en cuanto a número de asignaturas se refiere con respecto a las específicas de Publicidad y el planteamiento general de las mismas, que continúan confinadas claramente a un segundo plano, sigue poseyendo tintes mayoritariamente instrumentales (tácticos y no estratégicos).

La situación se constata idéntica a la descrita por Xifra en 2007, tanto en grados como en licenciaturas, y tanto en las universidades públicas como en las privadas.

\subsection{Nuevas investigaciones}

Este trabajo de investigación se plantea como un punto inicial de referencia para un trabajo de continuidad indefinida en el tiempo y de periodicidad anual, con la finalidad de mantener permanentemente actualizado el estado de la cuestión sobre la oferta docente de las universidades españolas, para informar a la "Cátedra Dircom.Cat" de los cambios de interés producidos, con la finalidad de incorporarlos al futuro Libro Blanco de Dircom Cataluña.

También se constituye en preludio de otra nueva investigación, en esta ocasión de carácter cualitativo, que pretende analizar los contenidos de las diversas asignaturas y módulos del 
mismo objeto de estudio, así como de las competencias de los estudiantes, y su posible evolución durante los próximos 5 años académicos (2010 a 2015).

\subsection{Conclusiones}

Recordemos que la unidad de análisis objeto de nuestra investigación está configurada por 30 universidades presenciales (de las cuales 15 son públicas y 15 son privadas), más 1 pública no presencial, lo que supone un total de 32 universidades españolas $(42,66 \%)$ que ofertan titulaciones de Grado/Licenciatura de Publicidad y Relaciones Públicas en el conjunto de las 75 universidades que configuran el sistema universitario del estado español -excluidas las dos especiales-.

Con respecto a la pregunta formulada: “¿Son mayoría (>50\%) los centros de enseñanza universitaria del estado español que ofertan conocimientos en Publicidad y Relaciones Públicas que han obtenido la verificación del grado en el curso 2009-10?", la respuesta es que no, ya que no se alcanza el $50 \%$.

Con respecto a las hipótesis planteadas, se refuta la $1^{\text {a }}$ hipótesis, ya que no son mayoría (>50\%) los grados que ofertan conocimientos en Publicidad y Relaciones Públicas que presentan modificaciones en la denominación del mismo en el curso 2009-10, ya que sólo ascienden a un total de $7(21,87 \%)$.

Asimismo se refuta la $2^{\text {a }}$ hipótesis, ya que no son mayoría (>50\%) los grados que ofertan conocimientos en Publicidad y Relaciones Públicas que han igualado el número de asignaturas de ambas áreas de conocimiento en el curso 2009-10. Es más, únicamente el Grado de la Universidad Cardenal Herrera Oria-CEU (campus de Moncada, Valencia) ha igualado en 5 las asignaturas del área de Relaciones Públicas y las del área de Publicidad y casi lo consigue la Escuela Superior de Relaciones Públicas-ESRP, centro adscrito a la Universidad de Barcelona que imparte el Grado en Publicidad y Relaciones Públicas (9 de Relaciones Públicas contra 10 de Publicidad).

\section{Bibliografía}

ADECEC - Asociación de Empresas Consultoras en Relaciones Públicas y Comunicación (2005 y 2008): La Comunicación y las Relaciones Públicas: Radiografía del sector, Barcelona: ADECEC. 
Agut, S. y Lozano, F.A. (2008): “Las competencias a debate: su papel en el proceso de convergencia europea en educación superior, Revista de Psicología General y Aplicada, 61, (1), pp. 53-68

ANECA - Agencia Nacional de Evaluación de la Calidad y Acreditación (2005): Títulos de Grado en Comunicación: Libro Blanco, Madrid: ANECA. http://www.aneca.es/ y

ANECA - Agencia Nacional de Evaluación de la Calidad y Acreditación (2008): Guía de Evaluación del diseño del Sistema de Garantía Interna de Calidad de la formación universitaria, Madrid: ANECA. http://www.aneca.es/media/166350/audit doc04 guiaevaluacion 080219.pdf

Bará, J. (2008): "El espacio europeo de garantía de la calidad en la educación superior", Coneixement i Societat. Revista d'Universitats, Recerca i Societat de la Informació, 15, pp. 6-37.

Botan, C. H. y Hazleton, V. (2006): "Public Relations in a New Age", en C. H. Botan y V. Hazleton (eds.), Public Relations Theory II, Mahwah (NJ): Erlbaum, pp. 1-18.

Botan, G. M., y Taylor, M. (2004): "Public Relations: State of the Field", Journal of Communication, (December), pp. 645-661

Broom, G. M. y Dozier, D. M. (1986). “Advancement for Public Relations Role Models”, Public Relations Review, 7 (1), pp. 37-56.

Caballero, Mํㅡㄹ A. (2007): "La adaptación al espacio europeo de Educación Superior como escenario para la reflexión sobre el proceso de enseñanza-aprendizaje en el ámbito universitario: algunas propuestas para un cambio significativo", Revista Española de Orientación y Psicopedagogía, 18, (2), pp. 167-177.

Campillo, M.; Pérez, M. y Gutiérrez, M. (2008): “Actores y campos: de lo científico y lo profesional", $\underline{\text { Red U. Revista de Docencia Universitaria, } 2 .}$

Cano, $M^{a}$ E. (2008), "La evaluación por competencias en la educación superior", Profesorado. Revista de Currículum y Formación del Profesorado, 12, (3).

Casado, R. (2006): "Convergencia con Europa y cambio en la universidad", EDUTEC Revista Electrónica de Tecnología Educativa, 20. 
Castillo, A. y Xifra, J. (2006): "Investigación bibliométrica de las tesis doctorales españolas sobre Relaciones Públicas (1965-2005)”, Anàlisi, 34, pp. 141-161.

Commission on Public Relations Education (2006): The Professional Bond - Public Relations Education and the Practice, NewYork: Public Relations Society of America. http://www.commpred.org/

Dozier, D. M. y Broom, G. M. (1995): "Evolution of the Manager Role in Public Relations Practice", Journal of Public Relations Research, 7 (1), pp. 3-26.

Escudero, J.M.; Vallejo, M. y Botías, F. (2008), "El asesoramiento en educación: ¿Podrían ser las competencias profesionales una contribución positiva?", Profesorado. Revista de Currículum y Formación del Profesorado, 12, (1).

Esteban, M. y Sáez, J. (2008), "Las profesiones, las competencias y el mercado", $\underline{R e d ~ U .}$ Revista de Docencia Universitaria, 2.

Fidalgo, R. y García Sánchez, J.N. (2007): "Las directrices del Espacio Europeo de Educación Superior en el Marco Legislativo del Sistema Universitario Español”, $\underline{\text { Aula }}$ Abierta, 35, (1-2), pp. 35-48.

García Aretio, L. y Ruiz Corbella, M. (2008): "Las instituciones de educación superior europeas: la presión por su modernización”, Innovación Educativa, 18, pp. 9-22.

García Gallego, A. y Blanco, P. (2007): "De La Sorbona a Londres: el camino hacia el Espacio Europeo de Educación Superior (adaptación en España: especial referencia a los Estudios Económico-Empresariales), Pecvnia. Revista de la Facultad de Ciencias Económicas y Empresariales, 5, pp. 107-144.

García Martínez, J. (2007): "El entrenamiento en competencias como foco del Espacio Europeo de Educación Superior", Perficit, 27, (1), pp. 93-114.

Gavari Starkie, E. (2009): "El papel del docente y del alumno en la elaboración de portafolios", Revista de Educación, (Madrid), 349, pp. 451-462.

González, M. y Raposo, M. (2009): "Valoraciones del profesorado universitario sobre las acciones que pueden favorecer el proceso de Convergencia Europea", Revista de Educación (Madrid), 349, pp. 361-390. 
González, M.; Muñoz, P. y Muñoz, M. (2008): "Análisis de las web específicas sobre EEES de las universidades españolas", Red. Revista de Educación a Distancia (Murcia), 19.

Haug, G. (2008): "La legislación europea y las legislaciones nacionales", Revista de Educación (Madrid), no Extraordinario 1, pp. 285-308

Hernández, S.; Losada, J.C., y Matilla, K. (2009): “Las Relaciones Públicas y la Comunicación Corporativa en la oferta universitaria de Postgrado del estado español: análisis sistemático y proyectivo y una iniciativa de observatorio del estado de la situación por parte de una asociación profesional", Razón y Palabra, no 70, Noviembre-Febrero, número monográfico sobre Relaciones Públicas Coordinado por la Dra. Yanyn Rincón Quintero.

Huertas, A., y Cavia, J. F. (2006): "Center and Periphery: Two Speeds for the Implementation of Public Relations in Spain”, Public Relations Review, 32, (2), pp. 110-117.

Juanas, A. de; Fernández, Mâ P. (2008): "Competencias y estrategias de aprendizaje. Reflexiones sobre el proceso de cambio en el EEES", Cuadernos de Trabajo Social, 21, pp. 217-230.

Karseth, B. (2006): "Reestructuración del Plan de Estudios en Enseñanza Superior después del Proceso de Bolonia: ¿un nuevo régimen pedagógico?”, Revista Española de Educación Comparada, número monográfico: "El Proceso de Bolonia. Dinámicas y desafíos de la enseñanza superior en Europa a comienzos de una nueva época", 12, pp. 255-284

L'Etang, J. y Pieczka, M. (2006): "Public Relations Education”, en J. L'Etang y M. Pieczca (eds.), Public Relations: Critical Debates and Contemporary Practice (pp. 433-442), Hillsdale (NJ): Erlbaum

Lalueza, F. (2009): "Lo que hay que tener. Estudio comparativo de las competencias requeridas al profesional de las Relaciones Públicas en el modelo formativo estadounidense y en el modelo formativo español post-Bolonia". Actas del IV Congreso Internacional de la AIRP - Asociación de Investigadores de Relaciones Públicas, Abril 2009, Barcelona: AIRP. 
León Duarte, G. A. (2007): "Estrategias y prácticas científicas de las revistas científicas latinoamericanas de la comunicación. Una aproximación a sus características estructurales", Revista Latina de Comunicación Social, 62.

Magallón, S. (1998): Definición empírico-operativa del «practicum» de los estudiantes universitarios de Relaciones Públicas y su vinculación con la realidad profesional en Cataluña, 1992-1997, Tesis Doctoral defendida en la Universidad Autónoma de Barcelona. http://www.tdx.cat/TDX-0622109-171218

Martín, E. (2009): "El marco europeo de cualificaciones para el aprendizaje permanente. Un instrumento para fomentar la movilidad laboral y la transparancia del mercado de trabajo", Relaciones Laborales. Revista Crítica de Teoría y Práctica, 12, pp. 105-115.

Martínez Lirola, M. (2008), "El uso del portafolio como herramienta metodológica y evaluadora en el proceso de convergencia europea", Profesorado. Revista de Currículum y Formación del Profesorado, 12, (2).

Martínez, P.; Martínez, M. y Muñoz, M. (2008), “Aprendizaje de competencias en educación superior", Revista Galego-Portuguesa de Psicoloxía e Educación, 16, (1-2), pp. 195-215

Heath, R. L., y Coombs, W. T. (2006). Today's Public Relations: An Introduction. Thousand Oaks (CA): Sage.

Martínez Mendoza, S. (2007): "En torno a la investigación en Relaciones Públicas", Revista Latina de Comunicación Social, 62, pp. 102-116.

Matilla, K. y Xifra, J. (2009): “Comunicación Corporativa y Relaciones Públicas: un proyecto de aproximación universidad/profesión en Cataluña, en el contexto de integración al Espacio Europeo de Enseñanza Superior", Razón y Palabra, no 70, Noviembre-Febrero, número especial monográfico sobre Relaciones Públicas coordinado por la Dra. Yanyn Rincón Quintero.

Michavila, F. y Parejo, J.L. (2008), "Políticas de participación estudiantil en el Proceso de Bolonia", Revista de Educación (Madrid), nº Extraordinario 1, pp. 85-118

Michavila, F. y Zamorano, S. (2008): "Panorama de los sistemas de garantía de calidad en Europa: una visión transnacional de la acreditación”, Revista de Educación (Madrid), no Extraordinario 1, pp. 235-266 
Ministerio de Educación (2006): Propuestas para la renovación de las metodologías educativas de la universidad, Madrid: Consejo de Coordinación Universitaria de la Secretaría de Estado de Universidades e Investigación. http://www.educacion.es/dctm/mepsyd/educacion/universidades/estadisticasinformes/estadisticas/propuestarenovacion.pdf?documentld=0901e72b80048b70

Ministerio de Educación (2009): Datos y cifras del Sistema Universitario. Curso 2009-10. Madrid: Secretaría General de Universidades, Subdirección General de Análisis, Estudios y Prospectiva Universitaria. http://www.educacion.es/dctm/ministerio/educacion/universidades/estadisticasinformes/datos-cifras/2009-datos-y-cifras-09-10.pdf?documentld=0901e72b8009f6bb

Ortega, J.L. y Aguillo, I. F. (2007): "La web académica española en el contexto del Espacio Europeo de Educación Superior: estudio exploratorio", El Profesional de la Información, 16, (5), pp. 417-425.

Pablos, J. de; Colás, Ma P.S.; González Ramírez, T. y Jiménez Cortés, R. (2007): "La adaptación de las universidades al Espacio Europeo de Educación Superior. Un procedimiento metodológico para el diseño de planes estratégicos", RIE. Revista Investigación Educativa, 25, (2), pp. 533-554.

Padilla, Ma T. y Gil, J. (2008), "La evaluación orientada al aprendizaje en la Educación Superior: condiciones y estrategias para su aplicación en la docencia universitaria", Revista Española de Pedagogía, 66, 241, pp. 467-485.

Pardo, J.C. y García Tobío, A. (2008): "El Espacio Europeo de Educación Superior: la razón instrumental de una universidad al servicio de la empresa", Innovación Educativa, 18, pp. $97-124$.

Pérez, A.; Tabernero, B.; López, V. M.; Ureña, N.; Ruiz, E.; Caplloch, M.; González, N. y Castejón, F.J. (2008), "Evaluación formativa y compartida en la docencia universitaria y el Espacio Europeo de Educación Superior: cuestiones clave para su puesta en práctica", Revista de Educación (Madrid), 347, pp. 435-451. 
Reques Velasco, P. (2007): "Presente y futuro de la enseñanza superior en España: algunos interrogantes abiertos", Perficit, 27, (1), pp. 115-130.

Rial, A. (2008): "Diseñar por competencias, un reto para los docentes universitarios en el Espacio Europeo de la Educación Superior", Innovación Educativa, 18, pp. 169-187.

Rodríguez Esteban, A. (2007): "Las competencias en el espacio europeo de educación superior: tipologías", Humanismo y Trabajo Social, 6, pp. 139-153

Rodríguez Izquierdo, R. Ma (2007): "Mejora continua de la práctica docente universitaria: una experiencia desde el proceso de convergencia del Espacio Europeo de Educación Superior", Revista Electrónica Interuniversitaria de Formación del Profesorado, 10, (1).

Sánchez, P. y Zubillaga, A. (2005): "Las universidades españolas ante el Proceso de Convergencia Europeo: análisis de las medidas institucionales y acciones de aplicación y coordinación", Revista de Educación (Madrid), 29, (337), pp. 169-187

Sallot, L. M.; Lyon, L. J.; Acosta-Alzuru, C. y Jones, K. O. (2003): "From Aardvark to Zebra: A New Millennium Analysis of Theory Development in Public Relations Academic Journals", Journal of Public Relations Research, 15 (1), p. 27-90.

Sanjosé, J. (2007): “La estrategia de Lisboa y la Declaración de Bolonia”, Perficit, 27, (1), pp. $9-28$

Seitel, F.P. (2002): Teoría y Práctica de las Relaciones Públicas, 8a ed, Prentice Hall: Madrid, (Seitel, F.P. (2001): The practice of Public Relations (8 $8^{\underline{a}}$ ed.). Upper Saddle River (NJ): Prentice Hall.

Tilson, D. J.y Pérez, P .S. (2003): "Public Relations and the New Golden Age of Spain: a Confluence of Democracy, Economic Development and the Media", Public Relations Review, 29, (2), pp. 125-143.

Vázquez, J. A. (2008): "La organización de las enseñanzas de grado y posgrado, $\underline{\text { Revista de }}$ Educación (Madrid), no Extraodinario 1, pp. 23-40.

Vázquez, J. A. (2007): "La universidad innovadora", Economistas, 25, (111), pp. 324-327. 
Velasco, F. (2005): "Diógenes como pretexto: una Ética para qué", Sociedad y Utopía, 26, pp. 57-69

Villa, A. (2008): "La excelencia docente", Revista de Educación (Madrid), Extraordinario 1, pp. 177-212

Wright, D.K., y Turk, J.V. (2007): "Public Relations Knowledge and Professionalism: Challenges to Educators and Practitioners", en E. L. Toth (ed.), The Future of Excellence in Public Relations and Communication Management: Challenges for the Next Generation, Mahwah, NJ: Erlbaum, pp. 571-588.

Xifra, J. (2007): “Undergraduate Public Relations Education in Spain: Endangered Species?”, Public Relations Review, 33, pp. 206-213.

Xifra, J., y Castillo, A. (2006): "Forty Years of Doctoral Public Relations Research in Spain: A Quantitative Study of Dissertation Contribution to Theory Development", Public Relations Review, 32, (3), pp. 302-308.

Zabalza Beraza, M.A. (2008): "El Espacio Europeo de Educación Superior: innovación en la enseñanza universitaria”, Innovación Educativa, 18, pp. 69-95.

\section{Autores}

Dra. Kathy Matilla, Departamento de Publicidad y Relaciones Públicas - Facultad de Comunicación Blanquerna, Universidad Ramón Llull (URL).

c/ Valldonzella, 22, 08001 Barcelona (Barcelona, España).

Teléfono: 607833849 - fax: - - -

Correo electrónico: kathyms@blanquerna.url.edu

Dr. Salvador Hernández, Departamento de Comunicación - Facultad de Ciencias Sociales y de la Comunicación.

Universidad Católica San Antonio (UCAM).

Campus de los Jerónimos, 30107 Guadalupe (Murcia, España).

Teléfono: 968278744 - fax: - - -

Correo electrónico: SHernandez@pdi.ucam.edu 
Dr. José Carlos Losada, Departamento de Comunicación - Facultad de Ciencias Sociales y de la Comunicación.

Universidad Católica San Antonio (UCAM)

Campus de los Jerónimos, 30107 Guadalupe (Murcia, España)

Teléfono: 968278170 - fax: - - -

Correo electrónico: jclosada@hotmail.com

* Este artículo muestra los resultados del proyecto de investigación llevado a cabo por encargo de la "Cátedra Dircom.Cat," de la Asociación de Directivos de Comunicación ADC Dircom Cataluña.

\section{Forma de citar este artículo en bibliografías}

Matilla, K.; Hernández, S. y Losada, C. (2010): "La oferta formativa de Relaciones Públicas en los nuevos Grados universitarios en España (2009-10)", en Revista PANGEA, 1, páginas 124 a 159. Red Académica Iberoamericana de Comunicación. Recuperado el _ de de 2 de: http://revistapangea.org/2010/12/05/01-01-107/ 